\title{
On the relations between ELECTRE TRI-B and ELECTRE TRI-C and on a new variant of ELECTRE TRI-B ${ }^{1}$
}

\author{
Denis Bouyssou ${ }^{2} \quad$ Thierry Marchant 3
}

22 September 2014

\begin{abstract}
ELECTRE TRI is a set of methods designed to sort alternatives evaluated on several criteria into ordered categories. The original method uses limiting profiles. A recently introduced method uses central profiles. We study the relations between these two methods. We do so by investigating if an ordered partition obtained with one method can also be obtained with the other method, after a suitable redefinition of the profiles. We also investigate a number of situations in which the original method using limiting profiles gives results that do not fit well our intuition. This leads us to propose a variant of ELECTRE TRI that uses limiting profiles. We show that this variant may have some advantages over the original method.
\end{abstract}

Keywords: Decision with multiple attributes, Sorting models, ELECTRE TRI-B, ELECTRE TRI-C.

\section{Introduction}

This paper deals with ELECTRE TRI. It consists in a set of methods that are the most recent ones belonging to the ELECTRE family of methods (for overviews, see Roy and Bouyssou, 1993, Ch. 5 \& 6, Figueira, Mousseau, and Roy, 2005, Figueira, Greco, Roy, and Słowiński, 2013).

ELECTRE TRI was originally introduced in the doctoral dissertation of Wei (1992) (supervised by B. Roy) and was detailed in Roy and Bouyssou (1993, p. 389-401). The original method is designed to sort alternatives evaluated on multiple criteria into ordered categories defined by limiting profiles (see Roy and Bouyssou, 1993, Ch. 6, for a detailed analysis of the sorting problem formulation). This method has generated much interest. Indeed, sorting alternatives into ordered categories is a problem occurring in many real-world situations. Moreover, on a more technical level, the fact that the method only compares alternatives with a set of carefully selected limiting profiles that are linked by dominance greatly facilitates the exploitation of the outranking relation that is built. This limits the consequences of the fact that this relation is, in general, neither transitive nor complete (Bouyssou, 1996). Many techniques have been proposed for the elicitation of the parameters of this method (see Cailloux, Meyer, and Mousseau, 2012, Damart, Dias, and Mousseau, 2007, Dias and

\footnotetext{
Authors are listed in alphabetical order. They have contributed equally to the paper.

${ }^{1}$ During the preparation of this paper, Thierry Marchant benefited from a visiting position at the Université Paris Dauphine. This support is gratefully acknowledged. Jose R. Figueira, Bernard Roy and anonymous referees of this journal made helpful comments on earlier drafts of this text. We are fully responsible for all remaining problems or errors.

${ }^{2}$ CNRS, LAMSADE, UMR 7243 \& Université Paris Dauphine, Place du Maréchal de Lattre de Tassigny, F-75775 Paris Cedex 16, France, tel: +33 1440548 98, fax: +33 1440540 91, e-mail: bouyssou@lamsade.dauphine.fr.

${ }^{3}$ Ghent University, H. Dunantlaan, 1, B-9000 Gent, Belgium tel: +32926463 73, fax: +329264 64 87, e-mail: thierry.marchant@UGent. be.
} 
Clímaco, 2000, Dias and Mousseau, 2003, 2006, Dias, Mousseau, Figueira, and Clímaco, 2002, Leroy, Mousseau, and Pirlot, 2011, Mousseau and Dias, 2004, Mousseau and Słowiński, 1998, Mousseau, Słowiński, and Zielniewicz, 2000, Mousseau, Figueira, and Naux, 2001, Mousseau, Figueira, Dias, Gomes da Silva, and Clímaco, 2003, Mousseau, Dias, and Figueira, 2006, Ngo The and Mousseau, 2002, Zheng, Metchebon Takougang, Mousseau, and Pirlot, 2014). Most of them use mathematical programming tools to infer the parameters of the method, based on assignment examples. This method has been applied to a large variety of real world problems (see the references at the end of Sect. 6 in Almeida-Dias, Figueira, and Roy, 2010). It has received a fairly complete axiomatic analysis in Bouyssou and Marchant (2007a,b). In a nutshell, ELECTRE TRI can be considered as a real success story within the ELECTRE family of methods.

A recent paper (Almeida-Dias et al., 2010) introduced a new method that uses central profiles instead of limiting profiles (Almeida-Dias et al., 2010, use the term "characteristic reference action" instead of central profiles). This is an interesting development since it seems "intuitively" easier to elicit central rather than limiting profiles (a related paper, Almeida-Dias, Figueira, and Roy, 2012, deals with the case of multiple central profiles. We do not study this more general case in the present paper).

The present paper was prompted by the analysis of this new method and its comparison with the original one. After having recalled the essential elements of both methods (Section 2), we investigate two main points.

We first study the relations between these two methods (Section 3). We do so by investigating if an ordered partition obtained with one method can also be obtained with the other method, after a suitable redefinition of the profiles. Our main conclusion is that this is not always possible. This fact should not be interpreted as a criticism of ELECTRE TRI but as the sign that the two methods that we study use, beyond surface, different principles.

We then present (Section 4) a number of situations in which the original method using limiting profiles gives results that do not fit well our intuition. These situations are mainly linked to the behavior of the method w.r.t. what we will call "strong dominance" and w.r.t. the transposition operation used by Almeida-Dias et al. (2010) to justify their proposal of two components of the method using central profiles that they recommend using conjointly. In particular, as first observed in Roy (2002), the two versions of the original method do not correspond via the application of this transposition operation: the pseudo-disjunctive version (also known as "optimistic") is not obtained from the pseudo-conjunctive version (also known as "pessimistic") via the transposition operation and vice versa. We detail this point that may explain why most of the elicitation techniques proposed so far only deal with the pseudo-conjunctive version (Zheng, 2012, Zheng et al., 2014, are exceptions) and why the axiomatic analysis conducted in Bouyssou and Marchant (2007a,b) is also limited to the pseudo-conjunctive version. This will lead us to propose a new variant of ELECTRE TRI using limiting profiles in which the two versions correspond via the transposition operation. We show that this new variant may have some advantages over the original method. A final section (Section 5) concludes with the indication of directions for future research ${ }^{1}$.

\footnotetext{
${ }^{1}$ In what follows, we will use the following terminology. ELECTRE TRI is a set of methods. ELECTRE TRI-B is a method that has two versions: ELECTRE TRI-B, pseudo-conjunctive, and ELECTRE TRI-B, pseudo-disjunctive. ELECTRE TRI-C is a method that has two components: ELECTRE TRI-C, ascending, and ELECTRE TRI-C, descending.

We sometimes abbreviate ELECTRE TRI-B, ELECTRE TRI-B, pseudo-conjunctive, and ELECTRE TRI-B, pseudodisjunctive as ETRI-B, ETRI-B-pc, and ETRI-B-pd.

We also sometimes abbreviate ELECTRE TRI-C, ELECTRE TRI-C, ascending, and ELECTRE TRI-C, descending as ETRI-C, ETRI-C-a, and ETRI-C-d.
} 


\section{ELECTRE TRI: a brief reminder}

We consider a set of alternatives $A$. Each alternative $a \in A$ is supposed to be evaluated on a family of $n$ real-valued criteria, i.e., $n$ functions $g_{1}, g_{2}, \ldots, g_{n}$ from $A$ into $\mathbb{R}$. These criteria are built with respect to a property $\mathcal{P}$. This property is usually taken to be "preference" but it can also be "riskiness" or "flexibility". Let us define $N=\{1,2, \ldots, n\}$. We suppose, w.l.o.g., that increasing the performance on any criterion increases the performance of an alternative w.r.t. the property $\mathcal{P}$. The dominance relation $\Delta$ is defined letting, for all $a, b \in A, a \Delta b$ if $g_{i}(a) \geq g_{i}(b)$, for all $i \in N$. In such a case, we say that $a$ dominates $b$. We say that a strictly dominates $b$ if $a \Delta b$ and $\operatorname{Not}[b \Delta a]$, which we denote by $a \Delta^{a} b$, since $\Delta^{a}$ is the asymmetric part of $\Delta$.

\subsection{Construction of the outranking relation}

In all the examples that follow, discordance will play no role and, on all criteria, the indifference and preference thresholds will be equal (hence, it is not restrictive to take them constant, see Roy and Vincke, 1987). In order to keep things simple, we briefly recall here how the outranking relation is built in this particular case. We refer, e.g., to Almeida-Dias et al. (2010, Section 2) or to Roy and Bouyssou (1993, p. 284-289) for the description of the construction of the outranking relation in the general case, i.e., when indifference and preference thresholds may be unequal and may vary and when discordance plays a role. It is important to realize the definition of the outranking relation that we detail below, although simpler than the general definition, is a particular case of the general one. All relations that can be obtained using the formulae in this section can be obtained using the more general formulae presented in Almeida-Dias et al. (2010, Section 2) and Roy and Bouyssou (1993, p. 284-289).

We associate with each criterion $i \in N$ a nonnegative preference threshold $p_{i} \geq 0$. If the value $g_{i}(a)-g_{i}(b)$ is positive but less than $p_{i}$, it is supposed that this difference is not significant, given the way $g_{i}$ has been built. Hence, on this criterion, the two alternatives should be considered indifferent.

The above information is used to define, on each criterion $i \in N$, a valued relation on $A$, i.e., a function from $A \times A$ into [0,1], called the partial concordance relation, such that:

$$
c_{i}(a, b)= \begin{cases}1 & \text { if } g_{i}(b)-g_{i}(a) \leq p_{i}, \\ 0 & \text { if } g_{i}(b)-g_{i}(a)>p_{i},\end{cases}
$$

(in the particular case studied here, the valued relations $c_{i}$ can only take the values 0 or 1 . In the general case, they can take any value between 0 and 1 ).

Each criterion $i \in N$ is assigned a non-negative weight $w_{i}$. We suppose, w.l.o.g., that weights have been normalized so that $\sum_{i=1}^{n} w_{i}=1$.

The valued relations $c_{i}$ are aggregated into a single valued outranking relation $s$ letting, for all $a, b \in A$,

$$
s(a, b)=\sum_{i=1}^{n} w_{i} c_{i}(a, b) .
$$

On the basis of the valued relation $s$, a binary relation $S_{\lambda}$ on $A$ is defined letting:

$$
a S_{\lambda} b \Leftrightarrow s(a, b) \geq \lambda,
$$

where $\lambda \in[0,1]$ is a cutting level (usually taken to be above $1 / 2$ ). The relation $S_{\lambda}$ is interpreted as saying "has at least as much of property $\mathcal{P}$ as" relation between alternatives (if, as is usually assumed, property $\mathcal{P}$ is taken to be "preference", the relation $S_{\lambda}$ is classically interpreted as an "at 
least as good as" relation between alternatives). From $S_{\lambda}$, we derive the following relations:

$$
\begin{aligned}
a P_{\lambda} b & \Leftrightarrow\left[a S_{\lambda} b \text { and } \operatorname{Not}\left[b S_{\lambda} a\right]\right], \\
a I_{\lambda} b & \Leftrightarrow\left[a S_{\lambda} b \text { and } b S_{\lambda} a\right], \\
a J_{\lambda} b & \Leftrightarrow\left[\operatorname{Not}\left[a S_{\lambda} b\right] \text { and } \operatorname{Not}\left[b S_{\lambda} a\right]\right],
\end{aligned}
$$

that are respectively interpreted as "has strictly more of property $\mathcal{P}$ as", "has as much of property $\mathcal{P}$ as", "is not comparable w.r.t. property $\mathcal{P}$ to" relations between alternatives (if property $\mathcal{P}$ is taken to be "preference", these relations are respectively interpreted as: "strictly better than", "indifferent to" and "incomparable to").

It is easy to check (Roy and Bouyssou, 1993, Ch. 5) that, if $a \Delta b$, then $s(a, b)=1$ and, for all $c \in A, s(b, c) \leq s(a, c)$ and $s(c, a) \leq s(c, b)$. Hence, if $a \Delta b$, we have $a S_{\lambda} b$ and, for all $c \in A$,

$$
\begin{aligned}
& b S_{\lambda} c \Rightarrow a S_{\lambda} c, \quad b P_{\lambda} c \Rightarrow a P_{\lambda} c, \\
& c S_{\lambda} a \Rightarrow c S_{\lambda} b, \quad c P_{\lambda} a \Rightarrow c P_{\lambda} b .
\end{aligned}
$$

The following proposition will be useful. It is taken from Roy and Bouyssou (1993, Ch. 6, p. 392-393) and its validity is independent of the simplifying hypotheses we have made concerning the construction of the outranking relation.

\section{Proposition 1}

Let $c^{1}, c^{2}, \ldots, c^{t} \in A$ be such that $c^{k+1} \Delta^{a} c^{k}$ for $k=1,2, \ldots, t-1$. Suppose furthermore that, for all $a \in A \backslash\left\{c^{1}, c^{t}\right\}, c^{t} P_{\lambda}$ a and $a P_{\lambda} c^{1}$.

When an alternative $a \in A \backslash\left\{c^{1}, c^{t}\right\}$ is compared to the subset of alternatives $c^{1}, c^{2}, \ldots, c^{t}$, three distinct situations may arise:

1. $c^{t} P_{\lambda} a, \ldots, c^{k_{1}+1} P_{\lambda} a, a P_{\lambda} c^{k_{1}}, a P_{\lambda} c^{k_{1}-1}, \ldots, a P_{\lambda} c^{1}$,

2. $c^{t} P_{\lambda} a, \ldots, c^{\ell_{2}+1} P_{\lambda} a, a I_{\lambda} c^{\ell_{2}}, a I_{\lambda} c^{\ell_{2}-1}, \ldots, a I_{\lambda} c^{k_{2}+1}, a P_{\lambda} c^{k_{2}}, \ldots, a P_{\lambda} c^{1}$,

3. $c^{t} P_{\lambda} a, \ldots, c^{\ell_{3}+1} P_{\lambda} a, a J_{\lambda} c^{\ell_{3}}, a J_{\lambda} c^{\ell_{3}-1}, \ldots, a J_{\lambda} c^{k_{3}+1}, a P_{\lambda} c^{k_{3}}, \ldots, a P_{\lambda} c^{1}$,

with $t>k_{1}>0, t>\ell_{2}>k_{2}>0$, and $t>\ell_{3}>k_{3}>0$.

The proof of the above proposition is quite easy (see Roy and Bouyssou, 1993, p. 392-393) and rests on the fact that the alternatives $c^{k}$ are linked by strict dominance and that the outranking relation is compatible with dominance.

\subsection{ELECTRE TRI-B}

The first method, called ELECTRE TRI in Roy and Bouyssou (1993, Ch. 6), uses limiting profiles. It was renamed ELECTRE TRI-B (ETRI-B, for short) by Almeida-Dias et al. (2010). We follow their naming conventions. ETRI-B has two versions called "pessimistic" and "optimistic" in Roy and Bouyssou (1993). Roy (2002) suggested, in view of the fact that property $\mathcal{P}$ may be something else than "preference", to rename the pessimistic version of the ELECTRE TRI-B as "pseudoconjunctive" (ETRI-B-pc, for short) and the optimistic version as "pseudo-disjunctive" (ETRI-B-pc, for short). This convention was followed in Almeida-Dias et al. (2010) and we do the same here.

We consider the case of $r$ ordered categories $C^{1}, C^{2}, \ldots, C^{r}$, with $C^{r}$ containing the alternatives having the more of property $\mathcal{P}$. The category $C^{k}$ is modelled using limiting profiles. The lower limiting profile of $C^{k}$ is $\pi^{k}$. The upper limiting profile of $C^{k}$ is $\pi^{k+1}$. Notice that the lower limiting profile $\pi^{k}$ of $C^{k}$ is also the upper limiting profile of $C^{k-1}$. Similarly, the upper limiting profile $\pi^{k+1}$ of $C^{k}$ is also the lower limiting profile of $C^{k+1}$ (see Figure 1). 


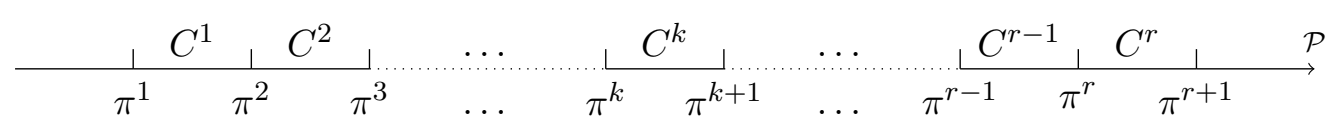

Figure 1: Sorting in ELECTRE TRI-B with $r$ ordered categories. The category $C^{r}$ contains the alternatives having the more of property $\mathcal{P}$. The category $C^{k}$ is defined by its lower limiting profile $\pi^{k}$ and its upper limiting profile $\pi^{k+1}$.

We suppose that the limiting profiles are such that $\pi^{k+1}$ strictly dominates $\pi^{k}$. The profile $\pi^{1}$ (resp. $\pi^{r+1}$ ) is taken to be arbitrarily low (resp. high). It will be convenient to suppose that $\pi^{k} \in A$, for $k=2,3, \ldots, r$, while $\pi^{1}, \pi^{r+1} \notin A$. With this convention, we have, for all $a \in A, a P_{\lambda} \pi^{1}$ and $\pi^{r+1} P_{\lambda} a$.

The two versions of ETRI-B are defined as follows (Roy and Bouyssou, 1993, p. 390-391).

\section{Definition 2 (ETRI-B-pc)}

Decrease $k$ from $r+1$ until the first value $k$ such that a $S_{\lambda} \pi^{k}$. Assign alternative a to $C^{k}$.

\section{Definition 3 (ETRI-B-pd)}

Increase $k$ from 1 until the first value $k$ such that $\pi^{k} P_{\lambda}$ a. Assign alternative a to $C^{k-1}$.

\section{Remark 4}

Take any $a \in A$. We know that $a P_{\lambda} \pi^{1}$ and $\pi^{r+1} P_{\lambda} a$. This implies that it is not true that $a S_{\lambda} \pi^{r+1}$ but that $a S_{\lambda} \pi^{1}$ holds. This shows that, with ETRI-B-pc, each alternative $a \in A$ is assigned to one of the categories $C^{1}, C^{2}, \ldots, C^{r}$.

Similarly, we know that $\pi^{r+1} P_{\lambda} a$ while we do not have $\pi^{1} P_{\lambda} a$. This shows that, with ETRI-B-pd, each alternative $a \in A$ is assigned to one of the categories $C^{1}, C^{2}, \ldots, C^{r}$.

\section{Remark 5}

Roy and Bouyssou (1993, Ch. 6, p. 393-395) have shown that if $a \in A$ is assigned to category $C^{k}$ by ETRI-B-pc and to category $C^{\ell}$ by ETRI-B-pd, then $k \leq \ell$. This explains why ETRI-B-pc (resp. ETRI-B-pd) was initially called pessimistic (resp. optimistic), with property $\mathcal{P}$ interpreted as "preference". Indeed, because of the definition of the profiles, we may apply Proposition 1 when comparing an alternative to the set of profiles $\pi^{1}, \pi^{2}, \ldots, \pi^{r+1}$. It is easy to check that the two versions of ETRI-B lead to identical results in the first (resp. second) case: $a$ is assigned to $C^{k_{1}}$ (resp. $C^{\ell_{2}}$ ), where $k_{1}$ (resp. $\ell_{2}$ ) is the highest index such that $a S_{\lambda} \pi^{k_{1}}$ (resp. a $S_{\lambda} \pi^{\ell_{2}}$ ). In the third case (i.e., when $\pi^{r+1} P_{\lambda} a, \ldots, \pi^{\ell_{3}+1} P_{\lambda} a, a J_{\lambda} \pi^{\ell_{3}}, \ldots, a J_{\lambda} \pi^{k_{3}+1}, a P_{\lambda} \pi^{k_{3}}, \ldots, a P_{\lambda} \pi^{1}$ ), ETRI-B-pc assigns $a$ to $C^{k_{3}}$ while ETRI-B-pd assigns it to $C^{\ell_{3}}$.

\section{Remark 6}

Definitions 2 and 3 are the ones found in Roy and Bouyssou (1993, p. 390-391). They require that categories are labelled in a way that is consistent with the orientation given by the property $\mathcal{P}$, the category $C^{r}$ (resp. $C^{1}$ ) containing the alternatives having the more (resp. the less) of property $\mathcal{P}$. These definitions are not well adapted to study the effect of the transposition operation, defined later. It will be useful to keep in mind the following equivalent definitions.

ETRI-B-pc assigns an alternative $a$ to the unique category $C^{k}$ such that $a$ is at least as good as to the lower limiting profile of this category and is not at least as good as its upper limiting profile (the relation "at least as good as" being $S_{\lambda}$ ).

ETRI-B-pd assigns an alternative $a$ to the unique category $C^{k}$ such that the upper limiting profile of this category is better than $a$ and the lower limiting profile of this category is not better than $a$ (the relation "better than" being $P_{\lambda}$ ). 


\subsection{ELECTRE TRI-C}

We still consider the case of $r$ ordered categories $C^{1}, C^{2}, \ldots, C^{r}$, with $C^{r}$ containing the alternative having the more of property $\mathcal{P}$. The category $C^{k}$ is modelled using a central profile $\omega^{k}$ (see Figure 2).

We suppose that the central profiles are such that $\omega^{k+1}$ strictly dominates $\omega^{k}$. Moreover, we adjoin to $\omega^{1}, \omega^{2}, \ldots, \omega^{r}$ two fictitious profiles $\omega^{0}$ and $\omega^{r+1}$. The profile $\omega^{0}$ (resp. $\omega^{r+1}$ ) is taken to be arbitrarily low (resp. high). It will be convenient to suppose that $\omega^{k} \in A$, for $k=1,2, \ldots, r$, while $\omega^{0}, \omega^{r+1} \notin A$. With this convention, we have, for all $a \in A, a P_{\lambda} \omega^{1}$ and $\omega^{r+1} P_{\lambda} a$.

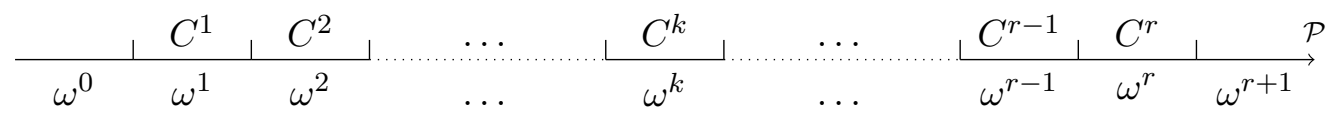

Figure 2: Sorting in ELECTRE TRI-C with $r$ ordered categories. The category $C^{r}$ contains the alternatives having the more of property $\mathcal{P}$. The category $C^{k}$ is defined by the central profile $\omega^{k}$.

For all $a, b \in A$, let $\rho(a, b)=\min (s(a, b), s(b, a)), \rho$ being called the selecting function in AlmeidaDias et al. (2010).

We define below the two components of ELECTRE TRI-C (ETRI-C, for short), called ELECTRE TRI-C, ascending (ETRI-C-a, for short) and ELECTRE TRI-C, descending (ETRI-C-d, for short), as introduced in Almeida-Dias et al. (2010). Almeida-Dias et al. (2010) recommend using these two components conjointly. This explains our choice of the term "component", instead of the term "version" that was used for ETRI-B.

\section{Definition 7 (ETRI-C-d)}

Decrease $k$ from $r+1$ until the first value $k$ such that $s\left(a, \omega^{k}\right) \geq \lambda$,

a) for $k=r$, assign a to $C^{r}$,

b) for $1 \leq k \leq r-1$, assign a to $C^{k}$ if $\rho\left(a, \omega^{k}\right)>\rho\left(a, \omega^{k+1}\right)$, otherwise assign a to $C^{k+1}$,

c) for $k=0$, assign a to $C^{1}$.

\section{Definition 8 (ETRI-C-a)}

Increase $k$ from 0 until the first value $k$ such that $s\left(\omega^{k}, a\right) \geq \lambda$,

a) for $k=1$, assign a to $C^{1}$,

b) for $2 \leq k \leq r$, assign a to $C^{k}$ if $\rho\left(a, \omega^{k}\right)>\rho\left(a, \omega^{k-1}\right)$, otherwise assign a to $C^{k-1}$,

c) for $k=r+1$, assign a to $C^{r}$.

These two components are to be used conjointly. This means that, if $a \in A$ is assigned to $C^{\ell}$ by one component of the method and to $C^{k}$ by the other one, the result of ETRI-C is that $a$ is assigned to the interval of categories having $C^{\ell}$ and $C^{k}$ as extremities. This interval may, of course, be reduced to a single category when $\ell=k$. Notice that, contrary to what is the case with ETRI-B, it is not true here that one component of the method always gives an assignment that is at least as high as the assignment given by the other component.

\section{Remark 9}

We know that $a P_{\lambda} \omega^{0}$ and $\omega^{r+1} P_{\lambda} a$. It is impossible that $s\left(a, \omega^{r+1}\right) \geq \lambda$. Moreover, we have $a S_{\lambda}$ $\omega^{0}$. Hence, each alternative $a \in A$ is assigned by ETRI-C-d to one of the categories $C^{1}, C^{2}, \ldots, C^{r}$.

Similarly, it is impossible that $s\left(\omega^{0}, a\right) \geq \lambda$. We also know that $\omega^{r+1} P_{\lambda} a$. Hence, each alternative $a \in A$ is assigned by ETRI-C-a to one of the categories $C^{1}, C^{2}, \ldots, C^{r}$. 


\section{Relations between ELECTRE TRI-B and ELECTRE TRI-C}

We have seen that Almeida-Dias et al. (2010) introduced the method ELECTRE TRI-C that aims at sorting alternatives between ordered categories using, for each category, a profile that is supposed to be "central", as opposed to the limiting profiles used in ELECTRE TRI-B (Roy and Bouyssou, 1993, Wei, 1992).

Because we have a method using limiting profiles (ETRI-B) and a method using central profiles (ETRI-C), a very natural question arises. Suppose that a result has been obtained using ETRI-B with limiting profiles. Between two consecutive limiting profiles, is it possible to find a central profile so that using ETRI-C with this family of central profiles, we obtain a similar result? Of course, the converse question may also be raised. Suppose that a result has been obtained using ETRI-C with central profiles. Between two consecutive central profiles, is it possible to find a limiting profile so that using ETRI-B with this family of limiting profiles, we obtain a similar result? These questions are not answered in Almeida-Dias et al. (2010) (Figueira, 2013, and Roy, 2013, reacting to a previous version of this text, indicate that this is because they felt that the two methods were really different. Almeida-Dias et al., 2010, sect. 6, study what happens if one applies ETRI-B using the profiles defined for ETRI-C keeping everything else unchanged).

ETRI-B has two versions. ETRI-C has two components. This leaves us with a total of eight questions to be answered. For instance, starting with an ordered partition obtained with ETRI-C-a is it always possible to modify the profiles, while keeping all other parameters unchanged (i.e., the preference and indifference thresholds, the weights, the cutting level, the ordering and number of categories), so that applying ETRI-B-pc to the same problem leads to the same ordered partition? (the reader might be perplexed by the fact that, in the statement of our problem, we have apparently ignored the recommendation of Almeida-Dias et al. (2010) to use the two components of ETRI-C conjointly. We have nevertheless taken care of this difficulty. In all the examples detailed below, the two components of ETRI-C will lead to the same result).

It turns out that the answer to these eight questions is negative. As already mentioned, we do not view this fact as a criticism of ETRI-C and/or ETRI-B but as an indication that the relations between ETRI-C and ETRI-B are complex. Despite sharing a common name and apparently being closely related, ETRI-B and ETRI-C seem to rest on somewhat different principles.

\subsection{From ELECTRE TRI-C to ELECTRE TRI-B, pseudo-conjunctive}

Because Bouyssou and Marchant (2007a,b) have given necessary and sufficient conditions for an ordered partition to be obtained with ETRI-B-pc, when preference and indifference thresholds are equal, this case is the easiest one. This also shows the power of axiomatic analysis for studying methods. Since all the examples used below will involve only two categories, we use only results from Bouyssou and Marchant (2007a).

Our aim is to establish the following proposition.

\section{Proposition 10}

There are ordered partitions that can be obtained with ETRI-C-a and that cannot be obtained with ETRI-B-pc, after a suitable redefinition of the profiles. The same conclusion holds with ETRI-C-d instead of ETRI-C-a.

\section{ProOF}

The proof consists in exhibiting suitable examples. Our first example has 6 criteria and 2 categories. For all criteria, in order to simplify the presentation, the preference and indifference thresholds are both null. The weights are denoted by $w_{i}$. We suppose that, on all criteria, the veto thresholds have been chosen so as to have no effect. We take $\lambda=0.6$. $H$ is the central profile of the High category $(\mathscr{H})$ and $L$ is the central profile of the Low category $(\mathscr{L})$. The main parameters that are used are 


\begin{tabular}{rrrrrrr}
\hline & $g_{1}$ & $g_{2}$ & $g_{3}$ & $g_{4}$ & $g_{5}$ & $g_{6}$ \\
\hline$w_{i}$ & 0.16 & 0.17 & 0.19 & 0.16 & 0.16 & 0.16 \\
$H$ & 75 & 75 & 75 & 75 & 75 & 75 \\
$L$ & 25 & 25 & 25 & 25 & 25 & 25 \\
\hline
\end{tabular}

Table 1: Main parameters in the example used the proof of Proposition 10.

\begin{tabular}{rrrrrrr}
\hline & $g_{1}$ & $g_{2}$ & $g_{3}$ & $g_{4}$ & $g_{5}$ & $g_{6}$ \\
\hline$a$ & 100 & 75 & 100 & 25 & 25 & 25 \\
$b$ & 50 & 100 & 100 & 50 & 25 & 25 \\
$c$ & 50 & 75 & 100 & 25 & 25 & 25 \\
$d$ & 0 & 100 & 100 & 50 & 25 & 25 \\
\hline
\end{tabular}

Table 2: Alternatives to be sorted $\mathrm{n}$ the example used the proof of Proposition 10.

presented in Table 1. The evaluation of the alternatives that are to be sorted are given in Table 2 . Applying ETRI-C to this example leads to the valued relation in Table 3.

Given that $\lambda=0.6$, we obtain with ETRI-C-d that

$$
a \in \mathscr{H}, b \in \mathscr{H}, c \in \mathscr{L}, d \in \mathscr{L} .
$$

Indeed, we have $s(a, H)=0.52<\lambda=0.6$ and $s(a, L)=1.0 \geq \lambda=0.6$. Alternative $a$ will be assigned to $\mathscr{H}$ if $\rho(a, H)=\min (s(a, H), s(H, a))>\rho(a, L)=\min (s(a, L), s(L, a))$. Using Table 3, we obtain $\rho(a, H)=0.52>\rho(a, L)=0.48$. It is easy to check that the same results are obtained using ETRI-C-a. For instance, we have $s(L, a)=0.48<\lambda=0.6$ and $s(H, a)=0.65 \geq \lambda=0.6$. Alternative $a$ will be assigned to $\mathscr{H}$ if $\rho(a, H)=\min (s(a, H), s(H, a))>\rho(a, L)=\min (s(a, L), s(L, a))$. Using Table 3, we obtain $\rho(a, H)=0.52>\rho(a, L)=0.48$.

Using the analysis in Bouyssou and Marchant (2007a), it is now easy to see that these assignments cannot be obtained using ETRI-B-pc. Indeed, it is shown in these papers (see Bouyssou and Marchant, 2007a, Lemma 20 \& Theorem 21, p. 230) that a necessary condition for assignments to be obtained with ETRI-B-pc, when the preference and indifference thresholds are equal and there is no veto involved, is that ${ }^{2}$ :

$$
\left.\begin{array}{c}
\left(x_{i}, \alpha_{-i}\right) \in \mathscr{H} \\
\text { and } \\
\left(y_{i}, \beta_{-i}\right) \in \mathscr{H}
\end{array}\right\} \Rightarrow\left\{\begin{array}{c}
\left(x_{i}, \beta_{-i}\right) \in \mathscr{H} \\
\text { or } \\
\left(z_{i}, \alpha_{-i}\right) \in \mathscr{H},
\end{array}\right.
$$

with the understanding that $\left(x_{i}, \alpha_{-i}\right)$ denotes the vector of evaluations of an alternative $e$ such that

\begin{tabular}{|c|c|c|c|c|c|c|}
\hline & $s(\cdot, H)$ & $s(H, \cdot)$ & $\rho(\cdot, H)$ & $s(\cdot, L)$ & $s(L, \cdot)$ & $\rho(\cdot, L)$ \\
\hline$a$ & 0.52 & 0.65 & 0.52 & 1.00 & 0.48 & 0.48 \\
\hline$b$ & 0.36 & 0.64 & 0.36 & 1.00 & 0.32 & 0.32 \\
\hline$c$ & 0.36 & 0.81 & 0.36 & 1.00 & 0.48 & 0.48 \\
\hline$d$ & 0.36 & 0.64 & 0.36 & 0.84 & 0.48 & 0.48 \\
\hline
\end{tabular}
$g_{i}(e)=x_{i}$ and $g_{j}(e)=\alpha_{j}$, for all $j \neq i$.

Table 3: Valued relation $s$ in the example used in the proof of Proposition 10. 
Taking $i=1, x_{i}=50, y_{i}=100, z_{i}=0, \alpha_{-i}=(100,100,50,25,25), \beta_{-i}=(75,100,25,25,25)$, we obtain :

$$
\begin{aligned}
& b=(50,100,100,50,25,25) \in \mathscr{H} \quad \text { and } \quad c=(50,75,100,25,25,25) \in \mathscr{L} \\
& a=(100,75,100,25,25,25) \in \mathscr{H} \quad \text { and } \quad d=(0,100,100,50,25,25) \in \mathscr{L} \text {. }
\end{aligned}
$$

This completes the proof.

The above proposition shows that there are assignments that can be obtained with ETRI-C-a (or ETRI-C-d) that cannot be obtained with ETRI-B-pc after a suitable redefinition of the profiles. In fact, the above proof shows more. Because the assignment obtained with ETRI-C-a (or ETRI-C-d) violates the necessary condition obtained in Bouyssou and Marchant (2007a, Lemma 20 \& Theorem 21 , p. 230), we could strengthen the above proposition allowing, not only for a change in the profiles, but also for a change of the indifference and preference thresholds (provided they remain equal, since the results in Bouyssou and Marchant (2007a) only cover this case), a change in the weights and a change in the cutting level $\lambda$. Indeed, in the conjoint measurement approach used in Bouyssou and Marchant (2007a), the condition exhibited above is necessary for a partition to be obtained with ETRI-B-pc, whatever the indifference and preference thresholds (provided they are equal), the weights, the cutting level and the limiting profile between $\mathscr{H}$ and $\mathscr{L}$.

Observe finally that, allowing for veto effects in ETRI-B-pc, would not change the conclusion of the above proposition, as long as we suppose, as in Bouyssou and Marchant (2007a), that veto effects occur in an all or nothing way, i.e., taking the valued relation to 0 as soon as there is a veto effect and keeping it unchanged otherwise. Indeed, in this case, a necessary condition (see Bouyssou and Marchant, 2007a, Theorem 35, p. 237, observing that the condition below is easily seen to be equivalent to the conjunction of conditions linear $_{i}$ and $3 v$-graded $_{i}$ ) for a partition to be obtained with ETRI-B-pc is that (using the notation presented in the above proof)

$$
\left.\begin{array}{l}
\left(x_{i}, \alpha_{-i}\right) \in \mathscr{H} \\
\text { and } \\
\left(z_{i}, \gamma_{-i}\right) \in \mathscr{H} \\
\text { and } \\
\left(y_{i}, \beta_{-i}\right) \in \mathscr{H}
\end{array}\right\} \Rightarrow\left\{\begin{array}{c}
\left(x_{i}, \beta_{-i}\right) \in \mathscr{H} \\
\text { or } \\
\left(z_{i}, \alpha_{-i}\right) \in \mathscr{H} .
\end{array}\right.
$$

Observing that ETRI-C-a and ETRI-C-d with the parameters used above lead to assign the alternative $(0,100,100,100,100,100)$ in category $\mathscr{H}$ (this alternative playing the role of $\left(z_{i}, \gamma_{-i}\right)$ in the above condition) shows that the impossibility to represent the information obtained using ETRI-B-pc is not due to the fact that an evaluation of 0 on $g_{1}$ prevents any alternative having such an evaluation from belonging to $\mathscr{H}$.

\subsection{From ELECTRE TRI-C to ELECTRE TRI-B, pseudo-disjunctive}

This is a more difficult case since the analysis in Bouyssou and Marchant (2007a,b) does not apply to ETRI-B-pd. Indeed, they have shown that the logic underlying ETRI-B-pd seems to be different from the one underlying ETRI-B-pc. This difference between the two versions of ETRI-B has also been observed in many works dedicated to the elicitation of parameters in ETRI-B that mainly concentrate on ETRI-B-pc.

Our aim is to establish the following proposition.

\section{Proposition 11}

There are ordered partitions that can be obtained with ETRI-C-a and that cannot be obtained with ETRI-B-pd, after a suitable redefinition of the profiles. The same conclusion holds with ETRI-C-d instead of ETRI-C-a. 
Proof

We consider the same example as in the proof of Proposition 10 and use the same notation. We have with both ETRI-C-a and ETRI-C-d,

$$
a \in \mathscr{H}, b \in \mathscr{H}, c \in \mathscr{L}, d \in \mathscr{L} .
$$

Let $\pi$ be the limiting profile between $\mathscr{H}$ and $\mathscr{L}$ used in ETRI-B-pd. Let us show that the only way to make the above assignments compatible with ETRI-B-pd is to choose $g_{1}(\pi)=50$.

With ETRI-B-pd, we have,

$$
\begin{aligned}
x \in \mathscr{H} & \Leftrightarrow \operatorname{Not}\left[\pi P_{\lambda} x\right], \\
x \in \mathscr{L} & \Leftrightarrow \pi P_{\lambda} x .
\end{aligned}
$$

We have

$$
\begin{aligned}
& b=(50,100,100,50,25,25) \in \mathscr{H} \\
& a=(100,75,100,25,25,25) \in \mathscr{H} \\
& c=(50,75,100,25,25,25) \in \mathscr{L} \\
& d=(0,100,100,50,25,25) \in \mathscr{L} .
\end{aligned}
$$

Hence, we know that

$$
\operatorname{Not}\left[\pi P_{\lambda}\left(x_{i}, \alpha_{-i}\right)\right] \text { and } \operatorname{Not}\left[\pi P_{\lambda}\left(y_{i}, \beta_{-i}\right)\right]
$$

together with

$$
\pi P_{\lambda}\left(x_{i}, \beta_{-i}\right) \text { and } \pi P_{\lambda}\left(z_{i}, \alpha_{-i}\right)
$$

Suppose that $\operatorname{Not}\left[\pi S_{\lambda}\left(x_{i}, \alpha_{-i}\right)\right]$ and $\operatorname{Not}\left[\pi S_{\lambda}\left(y_{i}, \beta_{-i}\right)\right]$. Because $\pi S_{\lambda}\left(z_{i}, \alpha_{-i}\right), \operatorname{Not}\left[\pi S_{\lambda}\right.$ $\left.\left(x_{i}, \alpha_{-i}\right)\right]$ implies that $\pi_{i}$ must be strictly below $x_{i}$ and greater than or equal to $z_{i}$. Because $\pi S_{\lambda}$ $\left(x_{i}, \beta_{-i}\right), \operatorname{Not}\left[\pi S_{\lambda}\left(y_{i}, \beta_{-i}\right)\right]$ implies that $\pi_{i}$ must be strictly below $y_{i}$ and greater than or equal to $x_{i}$. This is clearly impossible.

Suppose that $\left(x_{i}, \alpha_{-i}\right) S_{\lambda} \pi$ and $\left(y_{i}, \beta_{-i}\right) S_{\lambda} \pi$. Because $\left(x_{i}, \alpha_{-i}\right) S_{\lambda} \pi$ and $\operatorname{Not}\left[\left(z_{i}, \alpha_{-i}\right) S_{\lambda} \pi\right], \pi_{i}$ must be strictly above $z_{i}$ and less than or equal to $x_{i}$. Because $\left(y_{i}, \beta_{-i}\right) S_{\lambda} \pi$ and $\operatorname{Not}\left[\left(x_{i}, \beta_{-i}\right) S_{\lambda} \pi\right]$, $\pi_{i}$ must be strictly above $x_{i}$ and less than or equal to $y_{i}$. This is clearly impossible.

Suppose that $\operatorname{Not}\left[\pi S_{\lambda}\left(x_{i}, \alpha_{-i}\right)\right]$ and $\left(y_{i}, \beta_{-i}\right) S_{\lambda} \pi$. Because $\pi S_{\lambda}\left(z_{i}, \alpha_{-i}\right)$ and $\operatorname{Not}\left[\pi S_{\lambda}\right.$ $\left.\left(x_{i}, \alpha_{-i}\right)\right], \pi_{i}$ must be strictly below $x_{i}$ and greater than or equal to $z_{i}$. Because $\left(y_{i}, \beta_{-i}\right) S_{\lambda} \pi$ and $\operatorname{Not}\left[\left(x_{i}, \beta_{-i}\right) S_{\lambda} \pi\right], \pi_{i}$ must be strictly above $x_{i}$ and less than or equal to $z_{i}$. This is clearly impossible.

Suppose finally that $\left(x_{i}, \alpha_{-i}\right) S_{\lambda} \pi$ and $\operatorname{Not}\left[\pi S_{\lambda}\left(y_{i}, \beta_{-i}\right)\right]$ Because $\left(x_{i}, \alpha_{-i}\right) S_{\lambda} \pi$ and $\operatorname{Not}\left[\left(z_{i}, \alpha_{-i}\right) S_{\lambda}\right.$ $\pi], \pi_{i}$ must be strictly above $z_{i}$ and less than or equal to $x_{i}$. Because $\pi S_{\lambda}\left(x_{i}, \beta_{-i}\right), \operatorname{Not}\left[\pi S_{\lambda}\left(y_{i}, \beta_{-i}\right)\right]$ implies that $\pi_{i}$ must be strictly below $y_{i}$ and greater than or equal to $x_{i}$. This is the only possible case and we must have that $\pi_{i}$ is equal to $x_{i}=50$.

Let us now show that we must have $\pi_{i}=50$, for all $i \in N$. We already know that $\pi_{1}=50$. Because the weight of criteria $w_{1}=w_{4}=w_{5}=w_{6}=0.16$, it is clear, exchanging the roles of $g_{1}$ and $g_{i}$ with $i=4,5,6$, that the above example also shows that we must have $\pi_{1}=\pi_{4}=\pi_{5}=\pi_{6}=50$. The situation is slightly more difficult with $g_{2}$ and $g_{3}$ since their weights are different from the weight of $g_{1}$. Nevertheless the same example also works.

Indeed, for $g_{2}$, it suffices to consider the following alternatives:

\begin{tabular}{rrrrrrr}
\hline & $g_{1}$ & $g_{2}$ & $g_{3}$ & $g_{4}$ & $g_{5}$ & $g_{6}$ \\
\hline$a^{\prime}$ & 75 & 100 & 100 & 25 & 25 & 25 \\
$b^{\prime}$ & 100 & 50 & 100 & 50 & 25 & 25 \\
$c^{\prime}$ & 75 & 50 & 100 & 25 & 25 & 25 \\
$d^{\prime}$ & 100 & 0 & 100 & 50 & 25 & 25 \\
\hline
\end{tabular}


The reader will easily check that we have

$$
a^{\prime} \in \mathscr{H}, b^{\prime} \in \mathscr{H}, c^{\prime} \in \mathscr{L}, d^{\prime} \in \mathscr{L},
$$

with both ETRI-C-a and ETRI-C-d.

Similarly, for $g_{3}$, it suffices to consider the following alternatives:

\begin{tabular}{rrrrrrr}
\hline & $g_{1}$ & $g_{2}$ & $g_{3}$ & $g_{4}$ & $g_{5}$ & $g_{6}$ \\
\hline$a^{\prime \prime}$ & 100 & 75 & 100 & 25 & 25 & 25 \\
$b^{\prime \prime}$ & 100 & 100 & 50 & 50 & 25 & 25 \\
$c^{\prime \prime}$ & 100 & 75 & 50 & 25 & 25 & 25 \\
$d^{\prime \prime}$ & 100 & 100 & 0 & 50 & 25 & 25 \\
\hline
\end{tabular}

The reader will easily check that we have

$$
a^{\prime \prime} \in \mathscr{H}, b^{\prime \prime} \in \mathscr{H}, c^{\prime \prime} \in \mathscr{L}, d^{\prime \prime} \in \mathscr{L},
$$

with both ETRI-C-a and ETRI-C-d.

Hence, if the above partition (i.e., the one in which we have specified the assignment of $a, b$, $c, d, a^{\prime}, b^{\prime}, c^{\prime}, d^{\prime}, a^{\prime \prime}, b^{\prime \prime}, c^{\prime \prime}$, and $\left.d^{\prime \prime}\right)$ is to be represented using ETRI-B-pd, it must be true that $\pi=(50,50,50,50,50,50)$.

Let us finally show that this leads to a contradiction. Consider the alternative $e^{1}$ that has the evaluation 60 on all criteria except on a criterion 1 on which its evaluation is 25 . The reader will easily check that the alternative $e$ is assigned to $\mathscr{L}$ with both ETRI-C-a and ETRI-C-d. Since we now know the profile in ETRI-B-pd, it is easy to check that this leads to a contradiction. Indeed, we must have that $\pi P_{\lambda} e^{1}$. This implies that $\sum_{j \neq 1} w_{j}=0.84<\lambda=0.6$, a contradiction.

\section{Remark 12}

Considering, at the end of the above proof, not only $e^{1}$ but all the alternatives, $e^{i}, i=1,2, \ldots, 6\left(e^{i}\right.$ being the alternative evaluated at 60 on all criteria except on a criterion $i$ on which its evaluation is 25), it is easy to show that the contradiction remains even allowing for the use of a different set of weights and a different cutting levels in ETRI-B-pd. Indeed, we must have that $\pi P_{\lambda} e^{i}$, for all $i \in N$. This implies that, for all $i \in N$, we have:

$$
w_{i} \geq \lambda \text { and } \sum_{j \neq i} w_{j}<\lambda,
$$

a contradiction.

\subsection{From ELECTRE TRI-B to ELECTRE TRI-C}

This is also a difficult case since we do not have at hand an analysis similar to that of Bouyssou and Marchant (2007a,b) for ETRI-C. In order to keep things simple, we only analyze this case under the following two assumptions.

(a) If $\pi$ is the limiting profile used in ETRI-B to separate two categories, in ETRI-C the central profile of the higher category $\left(\omega^{k}\right)$ strictly dominates $\pi$ and $\pi$ strictly dominates the central profile of the lower category $\left(\omega^{k-1}\right)$. This seems an innocuous requirement that ensures a minimal semantic consistency between the profiles that are manipulated.

(b) We require that hyper-strict separability holds in the sense of Almeida-Dias et al. (2010, Condition 3 ), i.e., that, on all criteria, the central profile of the higher category is strictly preferred to the central profile of the lower category. Although this hypothesis is not completely necessary to prove the proposition below, it facilitates things greatly and appears to be quite reasonable. 


\begin{tabular}{rrrrrrrrrrrr}
\hline & $g_{1}$ & $g_{2}$ & $g_{3}$ & $g_{4}$ & $g_{5}$ & $g_{6}$ & $g_{7}$ & $g_{8}$ & $g_{9}$ & $g_{10}$ & $g_{11}$ \\
\hline$a$ & 0 & 0 & 0 & 0 & $10+\alpha$ & $10+\alpha$ & $10+\alpha$ & $10+\alpha$ & $10+\alpha$ & $10-\varepsilon$ & $10-\varepsilon$ \\
$\pi$ & 10 & 10 & 10 & 10 & 10 & 10 & 10 & 10 & 10 & 10 & 10 \\
\hline
\end{tabular}

Table 4: Example used the proof of Proposition 13. We have $\alpha, \varepsilon>0$.

Our aim is to establish the following proposition.

\section{Proposition 13}

Under the two assumptions ( $a$ ) and (b) made above, there are ordered partitions that can be obtained with ETRI-B-pc and that cannot be obtained with ETRI-C-a, after a suitable redefinition of the profiles. The same is true with ETRI-B-pd instead of ETRI-B-pc. The above two conclusions hold with ETRI-C-d, instead of ETRI-C-a.

\section{Proof}

Our example has 11 criteria and 2 categories. For all criteria, in order to simplify the presentation, the preference and indifference threshold are both null. The weight of all criteria is equal to $1 / 11$. We suppose that, on all criteria, the veto thresholds have been chosen so as to have no effect. The cutting level $\lambda$ is taken to be 0.52 , so that $1 / 2<\lambda<6 / 11$ and a coalition of 6 criteria among the set of 11 is necessary to obtain $S_{\lambda}$.

The limiting profile $\pi$ between the two categories is at 10 on all criteria. Consider now the alternative $a=(0,0,0,0,10+\alpha, 10+\alpha, 10+\alpha, 10+\alpha, 10+\alpha, 10-\varepsilon, 10-\varepsilon)$ with $\alpha, \varepsilon>0$ (see Table 4). We have $s(\pi, a)=6 / 11$ and $s(a, \pi)=5 / 11$. This implies $\pi P_{\lambda} a$. Hence, alternative $a$ is assigned to the lower category with either ETRI-B-pc or ETRI-B-pd.

We denote the central profile of the higher (resp. lower) category by $H$ (resp. $L$ ). Our hypotheses imply that, for all $i \in N$, we have $g_{i}(H)>10>g_{i}(L)$. Adjusting, if needed, the evaluation of $a$ on the first four criteria, it is easy to see that it is not restrictive to suppose that, for all $i \in N$, we have $g_{i}(L)>0$. Hence, we may choose $\alpha, \varepsilon$ to be such that $10+\alpha>g_{i}(H)>10, i=5,6,7,8,9$, and $10-\varepsilon>g_{j}(L)>0, j=10,11$. Whatever the choice of $g_{i}(H)$ and $g_{i}(L)$ compatible with our hypotheses, we obtain: $s(H, a)=6 / 11, s(a, H)=5 / 11, s(L, a)=4 / 11, s(a, L)=7 / 11$. Hence, with either ETRI-C-a or ETRI-C-d, alternative $a$ is assigned to the higher category. This completes the proof.

\section{A simple variant of ELECTRE TRI-B}

The present investigation, as well as our earlier work on ELECTRE TRI-B (see Bouyssou and Marchant, 2007a,b), has lead us to analyze closely the behavior of this method. We have been intrigued by a number of features of this method that we detail in Section 4.1. It turns out that it is possible to propose a simple variant ETRI-B that behaves more in accordance to our intuition w.r.t. these features. We present it in Section 4.2. The pros and cons of this simple variant are then analyzed in Section 4.3.

\subsection{Motivation}

\subsubsection{ELECTRE TRI-B and strong dominance}

Roy and Bouyssou (1993, p. 355) have proposed to analyze ETRI-B considering six "requirements": uniqueness (each alternative is assigned to a unique category), independence (the assignment of an alternative does not depend on the assignment of other alternatives), conformity (if $\pi^{k+1} P_{\lambda} a$ and $a P_{\lambda} \pi^{k}$ the alternative $a$ is assigned to $C^{k}$. Moreover, if $a I_{\lambda} \pi^{k}$, then $a$ is assigned to $C^{k}$ ), 
monotonicity (if $a \Delta b$, then $a$ is assigned to a category that has at least as much of property $\mathcal{P}$ than the one to which $b$ is assigned), homogeneity (alternatives comparing similarly to all profiles must be assigned to the same category), stability (the assignment of the alternatives should be consistent with the merging or the splitting of categories via the suppression of limiting profiles or the addition of new ones). Roy and Bouyssou (1993, p. 398-399) have shown that both ETRI-B-pc, and ETRI-B-pd, satisfy these six requirements, with the exception of the second part of the conformity requirement. Indeed, the second part of the conformity requirement asks that an alternative that is indifferent to the limiting profile $\pi^{k}$ should be assigned to $C^{k}$. However, it can happen that an alternative is indifferent to several consecutive profiles, in which case it is clearly impossible to satisfy the second part of the conformity requirement. Roy and Bouyssou (1993, p. 395) have introduced an additional condition, called "compatibility" requiring that if an alternative is indifferent to a profile, then it cannot be indifferent to the other profiles. With this additional condition, the second part of the conformity requirement is satisfied by both versions of ETRI-B. Roy and Bouyssou (1993, p. 396) nevertheless state that ETRI-B can still be used in case this additional condition does not hold.

Most of our discussion will be centered on the second part of the conformity requirement. At first sight, it seems perfectly sensible. Roy and Bouyssou (1993, p. 349-351) have motivated this condition considering the case of an assignment method that is based on the aggregation of the $n$ criteria into a single one via a function $V\left(g_{1}, g_{2}, \ldots, g_{n}\right)$. In such a case, in order to sort alternatives, one chooses $r+2$ thresholds $\lambda_{0}, \lambda_{1}, \lambda_{2}, \ldots, \lambda_{r}, \lambda_{r+1}$ such that $\lambda_{1}<\lambda_{2}<\cdots<\lambda_{r}$ and with $\lambda_{0}$ (resp. $\left.\lambda_{r+1}\right)$ arbitrarily low (resp. high). We can then use the following sorting rule:

$$
a \in C^{k} \Leftrightarrow \lambda_{k} \leq V\left(g_{1}(a), g_{2}(a), \ldots, g_{n}(a)\right)<\lambda_{k+1} .
$$

Clearly, with such a rule, the position of the non-strict inequality is purely conventional. The chosen convention is here to have all categories "closed below". This is the same convention that is at work in the second part of the conformity requirement. Once this convention has been settled, one can give a precise meaning to the profiles. They act as the lower limit of the categories, with the understanding that, at the lower limit, an alternative still belongs to the category.

The analogy with a method that is based on the aggregation of all criteria into one can be misleading however. In this situation, the case of an alternative $a \in A$ such that $V\left(g_{1}(a), g_{2}(a), \ldots, g_{n}(a)\right)$ is equal to one of the thresholds $\lambda_{k}$ is clearly exceptional (at least when the function $V$ is taken to be strictly increasing in all its arguments as, e.g., in the UTADIS method, see Greco, Mousseau, and Słowiński, 2010, Jacquet-Lagrèze, 1995, Zopounidis and Doumpos, 2000). Therefore, in this case, the convention of taking the categories to be closed below (or closed above) has hardly any practical consequences. This is not the case when using a preference model such as the one used in ETRI-B. Indeed, with the preference model used in ETRI-B, we cannot suppose anymore that indifference to a profile is exceptional and that the convention of taking categories to be closed below has no practical consequences. Indifference to a profile is not an exceptional case with the preference model used by ETRI-B.

This may lead to situations that are not easy to justify. Consider the following example. Alternatives are evaluated on 9 criteria (on a 0 to 100 scale) and are to be sorted into 2 ordered categories. Suppose that, on each criterion, the preference and indifference thresholds are both equal to 6 and that the veto thresholds have been chosen to be large enough so as to play no role. Suppose furthermore that all criteria have an equal weight and that $\lambda$ is taken to be 0.65 (so that a coalition of 6 criteria among the family of 9 criteria is necessary to obtain $S_{\lambda}$ ). If the limiting profile $\pi$ separating the two categories is at 50 on all criteria, we will conclude with both versions of ETRI-B that an alternative $a$ that is evaluated as $(45,45,45,45,45,45,30,30,30)$ will belong to the higher category since it is indifferent to the profile (we have: $s(\pi, a)=1$ and $s(a, \pi)=2 / 3$ ). This shows that indifference in the preference model used in ETRI-B can be quite "thick".

We say that a strongly dominates $b$ if $g_{i}(a)>g_{i}(b)$, for all $i \in N$, which we denote by $a \Delta^{*} b$. Because ETRI-B makes use of preference model in which indifference can be "thick", it may happen 
that an alternative $a$ is strongly dominated by $\pi^{k+1}$ and strongly dominates $\pi^{k}$, while it is indifferent to $\pi^{k+1}$ (for simplicity, we suppose that $\pi^{k+1}$ is the only profile to which $a$ is indifferent, so that $\pi^{k+2} P_{\lambda} a$ and $a P_{\lambda} \pi^{k}$ ). In this case, the alternative $a$ will be assigned to $C^{k+1}$ by both ETRI-B-pc and ETRI-B-pd. Although the assignment of $a$ to $C^{k+1}$ may be justified, our view is that, in such a situation, assigning $a$ to $C^{k}$ should not be discarded. The above remark about the "thickness" of indifference implies that both versions of ETRI-B may violate a requirement stating that

$$
\pi^{k+1} \Delta^{*} a \Delta^{*} \pi^{k} \Rightarrow a \in C^{k} .
$$

In our view, this is the sign that the price to pay for a strict adherence to the second part of the conformity requirement is quite high: the choice between categories closed below or closed above is not as conventional with the preference model used in ETRI-B as it is with other preference models.

Moreover, as detailed below, adhering to the second part of the conformity requirement also implies that the two versions of ETRI-B do not correspond via the transposition operation.

\subsubsection{ELECTRE TRI-B and transposition}

We have seen that ETRI-C comes as the association of two components: ETRI-C-a and ETRI-C-d. Almeida-Dias et al. (2010) have proposed a very clever argument to justify the need for these two components and the necessity to use them conjointly. It is based on the transposition operation. This operation consists in inverting the direction of preference on all criteria and in inverting the ordering of the categories. Clearly, the conclusions obtained after this transposition operation should be the same as the original conclusions (provided that these new conclusions are reinterpreted with the original ordering of categories). Almeida-Dias et al. (2010, p. 569, bottom of 2 nd col.) indicate that the initial problem and the transposed problem are "equivalent". In the same vein, but this time referring to ETRI-B, Roy (2002, p. 13) indicates that the direction in which preference increases on the criteria as well as the ordering of the categories results from a "convention" and that "nothing prevents one from using the opposite convention" (our translation from Roy, 2002, p. 13, line -7). This is at the same time fairly intuitive and rather compelling. Hence, it seems advisable to analyze what happens when we use a sorting method before and after the transposition operation is applied. We do so for ETRI-B in this section. Indeed, if the argument is considered compelling when applied to ETRI-C, it is hard to imagine why it would not be so for ETRI-B. Roy (2002, pp. 13-14) agrees on this point since he explicitly analyzes, although briefly, the consequences of applying the transposition operation to ETRI-B.

Technically, the effect of the transposition operation is to transform $s(a, b)$ into $s(b, a)$ and vice versa. Almeida-Dias et al. (2010) have shown that the two components of ETRI-C correspond via the transposition operation. It is indeed easy to check that applying ETRI-C-a to a problem after it has been transposed amounts to applying ETRI-C-d, to the original problem. Similarly, applying ETRI-C-d to a problem after it has been transposed amounts to applying ETRI-C-a to the original problem. This gives very good grounds to justify the proposal of two components of ETRI-C and to require that they should be used conjointly.

In a regrettably unpublished paper, Roy (2002) was the first to observe that the two versions of ETRI-B do not correspond via the transposition operation: the pseudo-disjunctive version is not obtained from the pseudo-conjunctive version via the transposition operation and vice versa. He nevertheless observed that when applying the transposition operation to ETRI-B-pc one obtains a method that is "close" to ETRI-B-pd and vice versa.

This is easily explained. Remember from Section 2 that, because of the definition of the profiles, Proposition 1 applies when we compare an alternative to the set of profiles $\pi^{1}, \pi^{2}, \ldots, \pi^{r+1}$. We are in one of the three situations described in Table 5.

The category $C^{k}$ is limited below by $\pi^{k}$ and above by $\pi^{k+1}$. It is easy to check that the two versions of ETRI-B lead to identical results in the first (resp. second) case: $a$ is assigned to $C^{k_{1}}$ 


\begin{tabular}{ll}
\hline 1 & $\pi^{r+1} P_{\lambda} a, \ldots, \pi^{k_{1}+1} P_{\lambda} a, a P_{\lambda} \pi^{k_{1}}, a P_{\lambda} \pi^{k_{1}-1}, \ldots, a P_{\lambda} \pi^{1}$ \\
2 & $\pi^{r+1} P_{\lambda} a, \ldots, \pi^{\ell_{2}+1} P_{\lambda} a, a I_{\lambda} \pi^{\ell_{2}}, a I_{\lambda} \pi^{\ell_{2}-1}, \ldots, a I_{\lambda} \pi^{k_{2}+1}, a P_{\lambda} \pi^{k_{2}}, \ldots, a P_{\lambda} \pi^{1}$ \\
3 & $\pi^{r+1} P_{\lambda} a, \ldots, \pi^{\ell_{3}+1} P_{\lambda} a, a J_{\lambda} \pi^{\ell_{3}}, a J_{\lambda} \pi^{\ell_{3}-1}, \ldots, a J_{\lambda} \pi^{k_{3}+1}, a P_{\lambda} \pi^{k_{3}}, \ldots, a P_{\lambda} \pi^{1}$ \\
\hline
\end{tabular}

Table 5: The three cases occurring in comparing an alternative $a$ to the profiles $\pi^{1}, \pi^{2}, \pi^{3}, \ldots, \pi^{r}$, $\pi^{r+1}$.

(resp. $C^{\ell_{2}}$ ). In the third case, ETRI-B-pc assigns $a$ to $C^{k_{3}}$, while ETRI-B-pd assigns it to $C^{\ell_{3}}$.

After the transposition operation, the category $C^{k}$ is limited below by $\pi^{k+1}$ and above by $\pi^{k}$ (see Figure 3). Table 5 is turned into Table 6 after the transposition operation, where we use the symbols $\widehat{S}_{\lambda}, \widehat{P}_{\lambda}, \widehat{I}_{\lambda}$, and $\widehat{J}_{\lambda}$ instead of $S_{\lambda}, P_{\lambda}, I_{\lambda}$, and $J_{\lambda}$ to make clear that we are now working on a transposed problem. It is easy to check, using Remark 6 , that, on the transposed problem, the two versions of ETRI-B lead to identical results in the first (resp. second) case: $a$ is assigned to $C^{k_{1}}$ $\left(\right.$ resp. $C^{k_{2}}$ ). In the third case, ETRI-B-pc assigns $a$ to $C^{\ell_{3}}$ while ETRI-B-pd assigns it to $C^{k_{3}}$.

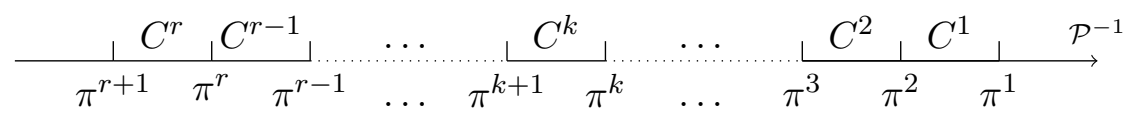

Figure 3: Sorting in ELECTRE TRI-B with $r$ ordered categories after transposition. The category $C^{1}$ contains the alternatives having the more of property $\mathcal{P}^{-1}$. The category $C^{k}$ is defined by its lower limiting profile $\pi^{k+1}$ and its upper limiting profile $\pi^{k}$.

\begin{tabular}{ll}
\hline 1 & $\pi^{1} \widehat{P}_{\lambda} a, \ldots, \pi^{k_{1}-1} \widehat{P}_{\lambda} a, \pi^{k_{1}} \widehat{P}_{\lambda} a, a \widehat{P}_{\lambda} \pi^{k_{1}+1}, \ldots, a \widehat{P}_{\lambda} \pi^{r+1}$ \\
2 & $\pi^{1} \widehat{P}_{\lambda} a, \ldots, \pi^{k_{2}} \widehat{P}_{\lambda} a, a \widehat{I}_{\lambda} \pi^{k_{2}+1}, \ldots, a \widehat{I}_{\lambda} \pi^{\ell_{2}-1}, a \widehat{I}_{\lambda} \pi^{\ell_{2}}, a \widehat{P}_{\lambda} \pi^{\ell_{2}+1}, \ldots, a \widehat{P}_{\lambda} \pi^{r+1}$ \\
3 & $\pi^{1} \widehat{P}_{\lambda} a, \ldots, \pi^{k_{3}} \widehat{P}_{\lambda} a, a \widehat{J}_{\lambda} \pi^{k_{3}+1}, \ldots, a \widehat{J}_{\lambda} \pi^{\ell_{3}-1}, a \widehat{J}_{\lambda} \pi^{\ell_{3}}, a \widehat{P}_{\lambda} \pi^{\ell_{3}+1}, \ldots, a \widehat{P}_{\lambda} \pi^{r+1}$ \\
\hline
\end{tabular}

Table 6: The three cases occurring when comparing an alternative $a$ to the profiles $\pi^{1}, \pi^{2}, \pi^{3}, \ldots$, $\pi^{r}, \pi^{r+1}$, after transposition.

The assignments that we obtain before transposition and after transposition are summarized in Table 7. It clearly shows that the two versions of ETRI-B do not correspond via the transposition operation: the pseudo-disjunctive version is not obtained from the pseudo-conjunctive version via the transposition operation and vice versa. This is due to the assignments resulting in case 2, the case in which we make use of the second part of the conformity requirement.

If we agree with the argument of Roy (2002) and Almeida-Dias et al. (2010) stating that the original and the transposed problem are "equivalent", this seems problematic. The convention taken on the orientation of the criteria has an influence on the results. This is not surprising: if a method satisfies the second part of the conformity requirement, applying the transposition operation leads to a method in which categories are closed above instead of being closed below. This conflict was first observed in Roy (2002). He concludes in favor of the satisfaction of the second part of conformity requirement rather than with in favor of the consistency with respect to transposition operation. Although Roy (2002) is brief on this point, we suspect that this has mainly to do with the definition of the profiles. In order to define the profiles, it is clearly useful to know to what category an alternative that is exactly identical to the profile should belong. While recognizing the strength of this argument, we consider the conflict between the second part of the conformity requirement and the transposition operation to be troublesome. We will propose below a solution to this problem that is different from that of Roy (2002), consisting in keeping ETRI-B as it was defined in Roy and Bouyssou (1993). 


\begin{tabular}{ccccc}
\hline & \multicolumn{2}{c}{ Before } & \multicolumn{2}{c}{ After } \\
& ETRI-B-pc & ETRI-B-pd & ETRI-B-pc & ETRI-B-pd \\
\hline 1 & $k_{1}$ & $k_{1}$ & $k_{1}$ & $k_{1}$ \\
2 & $\ell_{2}$ & $\ell_{2}$ & $k_{2}$ & $k_{2}$ \\
3 & $k_{3}$ & $\ell_{3}$ & $\ell_{3}$ & $k_{3}$ \\
\hline
\end{tabular}

Table 7: ETRI-B-pc, ETRI-B-pd before and after transposition

\subsubsection{Interpreting the two versions of ELECTRE TRI-B}

When ETRI-B was proposed (Roy and Bouyssou, 1993, Wei, 1992), its two versions (ETRI-B-pc and ETRI-B-pd) were not justified by appealing to the transposition operation. As their names suggest, the main argument was that, when comparing an alternative to a profile, one may wish to do so in a more or less conjunctive or disjunctive fashion.

Let us first recall, following Roy and Bouyssou (1993, p. 353-354), the definition of the conjunctive and disjunctive rules. With the conjunctive rule, an alternative $a$ is assigned to the unique category $C^{k}$ such that $a \Delta \pi^{k}$ and $\operatorname{Not}\left[a \Delta \pi^{k+1}\right]$. With the disjunctive rule, an alternative $a$ is assigned to the unique category $C^{k}$ such that $\pi^{k+1} \Delta a$ and $\operatorname{Not}\left[\pi^{k} \Delta a\right]$.

When preference and indifference thresholds are null and when $\lambda=1$, ETRI-B-pc is exactly equivalent with the conjunctive rule (Roy and Bouyssou, 1993, p. 397). Still using null preference and indifference thresholds and $\lambda=1$, ETRI-B-pd is close, but not exactly equivalent, to the disjunctive rule (Roy and Bouyssou, 1993, p. 397). When $a$ is exactly identical to $\pi^{k}$, the disjunctive rule will then assign $a$ to $C^{k-1}$, whereas ETRI-B-pd assigns it to $C^{k}$.

ETRI-B-pd and the disjunctive rule become equivalent, in the particular case in which indifference and preference thresholds are null and $\lambda=1$, if the disjunctive rule is modified as follows: an alternative $a$ is assigned to the unique category $C^{k}$ such that $\pi^{k+1} \Delta^{a} a$ and $\operatorname{Not}\left[\pi^{k} \Delta^{a} a\right]$, consisting in replacing the dominance relation $\Delta$ by the strict dominance relation $\Delta^{a}$ (Roy and Bouyssou, 1993, p. 397). Again, this is due to the willingness to stick to the second part of the conformity principle.

Notice that the conjunctive and disjunctive rules correspond via the transposition operation. Using a conjunctive rule on the original problem is equivalent to using the disjunctive rule on the transposed problem and vice versa. This correspondence is obtained abandoning the second part of the conformity requirement. The limiting profile $\pi^{k}$ is seen as a "frontier", i.e., it belongs to $C^{k}$ for the conjunctive rule and to $C^{k-1}$ for the disjunctive rule.

This also explains why the assignment rule in ETRI-B-pc is based on the relation $S_{\lambda}$, while the assignment rule of ETRI-B-pd uses $P_{\lambda}$. Considering that the preference model used in ETRI-B focuses on $S_{\lambda}$ and not on $P_{\lambda}$, we see that the desire to stick with the second part of the conformity principle has many consequences. Indeed, Bouyssou and Pirlot $(2009,2013,2014)$ have shown that the properties of $P_{\lambda}$ are significantly different from the ones of $S_{\lambda}$. They are also far more complex and difficult to analyze.

\subsubsection{Using the two versions of ELECTRE TRI-B}

Going through Roy and Bouyssou (1993), it is not completely clear whether or not they recommend to use the two versions of ETRI-B conjointly. In the words, in French, of Roy and Bouyssou (1993, p. 390), ETRI-B is the "jumelage" (i.e., "twinning") of ETRI-B-pc and ETRI-B-pd. This leaves room for different interpretations.

The later literature on ETRI-B has concentrated on ETRI-B-pc and the two versions of ETRI-B are not, in general, used conjointly. Most of the real-world applications of ETRI-B only use ETRI-B-pc (see the references at the end of Sect. 6 in Almeida-Dias et al., 2010). ETRI-B-pc is the only version 
that has received an axiomatic analysis (see Bouyssou and Marchant, 2007a,b). It is also the only one for which elicitation techniques have been widely developed (see Cailloux et al., 2012, Damart et al., 2007, Dias and Clímaco, 2000, Dias and Mousseau, 2003, 2006, Dias et al., 2002, Leroy et al., 2011, Mousseau and Dias, 2004, Mousseau and Słowiński, 1998, Mousseau et al., 2000, 2001, 2003, Ngo The and Mousseau, 2002).

We see this concentration of the literature on only one of the two versions of ETRI-B as an impoverishment. Concentrating on ETRI-B-pc and ignoring ETRI-B-pd one forgets that what is "conjunctive" when using the original problem becomes "disjunctive" with the, equivalent, transposed problem.

In order to justify the sole use of ETRI-B-pc, one may argue that the definition of the profiles should guide whether one wishes to use ETRI-B-pc or ETRI-B-pd. Although this is an interesting argument, we do not find it absolutely convincing. It is not explicitly used in Roy and Bouyssou (1993). On the contrary, they analyze (Roy and Bouyssou, 1993, p. 395) the relation between the assignments obtained with ETRI-B-pc and ETRI-B-pd, an analysis that would clearly be meaningless if different profiles should be used with ETRI-B-pc and ETRI-B-pd. Roy and Bouyssou (1993, p. 399, line -4) nevertheless state that the choice between ETRI-B-pc and ETRI-B-pd "can influence the choice of the profiles" (our translation and emphasis) but also state (p. 399, line 18) that "the exact position of the profiles cannot, except in exceptional cases, be rigorously deduced from the idea that one has of the categories" (our translation). Combining these two quotes, we find it difficult to accept the view that using ETRI-B-pc instead of ETRI-B-pd implies a complete redefinition of the profiles.

Our view is that the duality between the conjunctive and the disjunctive rule, using the same profiles, can profitably be maintained with ETRI-B. This is an incentive to use ETRI-B-pc and ETRI-B-pd conjointly. Yet, we have seen above that this conjoint use does not lead to results that correspond via the transposition operation.

\subsection{A simple variant of ELECTRE TRI-B}

We first introduce two new versions of ELECTRE TRI-B called dual of pseudo-conjunctive and dual of pseudo-disjunctive (often abbreviated as ETRI-B-dpc and ETRI-B-dpd).

\section{Definition 14 (ELECTRE TRI-B, dual of pseudo-conjunctive)}

Increase $k$ from 1 until the first value $k$ such that $\pi^{k} S_{\lambda}$ a. Assign alternative a to $C^{k-1}$.

\section{Definition 15 (ELECTRE TRI-B, dual of pseudo-disjunctive)}

Decrease $k$ from $r+1$ until the first value $k$ such that a $P_{\lambda} \pi^{k}$. Assign alternative a to $C^{k}$.

\section{Remark 16}

The above two definitions were conceived so as to be easily compared with Definitions 2 and 3. As was the case above (see Remark 6), they require that categories are labelled in a way that is consistent with the orientation given by the property $\mathcal{P}$, the category $C^{r}$ (resp. $C^{1}$ ) containing the alternatives having the more (resp. the less) of property $\mathcal{P}$. These definitions are therefore not well adapted to study the effect of the transposition operation, defined later. It will be useful to keep in mind the following equivalent definitions.

ETRI-B-dpc assigns an alternative $a$ to the unique category $C^{k}$ such that its upper limiting profile is at least as good as $a$ and its lower limiting profile is not at least as good as $a$ (the relation "at least as good as" being $S_{\lambda}$ ).

ETRI-B-dpd assigns an alternative $a$ to the unique category $C^{k}$ such that $a$ is better than its lower limiting profile and $a$ is not better than its upper limiting profile (the relation "better than" being $P_{\lambda}$ ). 
It is easy to check that ETRI-B-pc, and ETRI-B-dpc, correspond via the transposition operation. The same is true for ETRI-B-pd, and ETRI-B-dpd. The two variants ETRI-B-dpc, and ETRI-B-dpd just introduced obviously share the same properties as the original versions of ETRI-B, except that for the second part of the conformity principle. With these two variants, categories are now closed above. This is detailed in Tables 8 and 9. As explained above, if we agree with the arguments of Almeida-Dias et al. (2010) and Roy (2002) about the fact that the transposition operation transforms a problem into an equivalent one, and if we are prepared to accept the results of ETRI-B-pc (resp. ETRI-B-pd), we should also be prepared to accept the results of ETRI-B-dpc (resp. ETRI-B-dpd).

\begin{tabular}{ccccc}
\hline & \multicolumn{2}{c}{ Before } & \multicolumn{2}{c}{ After } \\
& ETRI-B-pc & ETRI-B-dpc & ETRI-B-pc & ETRI-B-dpc \\
\hline 1 & $k_{1}$ & $k_{1}$ & $k_{1}$ & $k_{1}$ \\
2 & $\ell_{2}$ & $k_{2}$ & $k_{2}$ & $\ell_{2}$ \\
3 & $k_{3}$ & $\ell_{3}$ & $\ell_{3}$ & $k_{3}$ \\
\hline
\end{tabular}

Table 8: ETRI-B-pc, ETRI-B-dpc, before and after transposition.

\begin{tabular}{ccccc}
\hline & \multicolumn{2}{c}{ Before } & \multicolumn{2}{c}{ After } \\
& ETRI-B-pd & ETRI-B-dpd & ETRI-B-pd & ETRI-B-dpd \\
\hline 1 & $k_{1}$ & $k_{1}$ & $k_{1}$ & $k_{1}$ \\
2 & $\ell_{2}$ & $k_{2}$ & $k_{2}$ & $\ell_{2}$ \\
3 & $\ell_{3}$ & $k_{3}$ & $k_{3}$ & $\ell_{3}$ \\
\hline
\end{tabular}

Table 9: ETRI-B-pd, ETRI-B-dpd, before and after transposition.

Our proposal is the following simple variant of ETRI-B. It consists in using ETRI-B-pc and ETRI-B-dpc conjointly ${ }^{3}$.

The pros and cons of this simple variant are analyzed below. Provided that one is prepared to abandon the second part of the conformity requirement, our proposal behaves more in accordance to our intuition w.r.t. the features presented in Section 4.1.

\subsection{Analysis of the variant of ELECTRE TRI-B}

Our proposal is simple. Basically, it amounts to abandon the second part of the conformity requirement so as to obtain results that correspond via the transposition operation. Hence, although our starting point is similar to Roy (2002), our conclusion is different.

Let us first observe, in line with Roy (2002), that our proposal remains "close" to the original ETRI-B. We modify it in two ways. First, we propose two versions that we suggest to use conjointly. Second, we abandon the second part of the conformity requirement.

If ETRI-B is interpreted as the conjoint use of ETRI-B-pc and ETRI-B-pd, our proposal only modifies the results of ETRI-B in the case an alternative is indifferent to one or several profiles. In

\footnotetext{
${ }^{3}$ We could have instead chosen to use ETRI-B-pd and ETRI-B-dpd conjointly. Our choice is motivated here by the fact that the construction of the outranking relation focuses on an "at least as good as" relation instead of a "strictly better than" one. This is also in line with the analysis in Bouyssou and Marchant (2007a,b). Both ETRI-B-pc and ETRI-B-dpc are defined using $S_{\lambda}$. Notice however that there is an argument in favor of the conjoint use of ETRI-B-pd and ETRI-B-dpd. As shown in Table 9, assignments with ETRI-B-dpd are always lower than the assignments with ETRI-B-pd. Such a choice would therefore maintain the relation between the assignments of ETRI-B-pc and ETRI-B-pd that obtains in ETRI-B. Notice that the conjoint use of ETRI-B-pd and ETRI-B-dpd would lead to the same intervals as the ones obtained in Table 10.
} 
ETRI-B, both ETRI-B-pd and ETRI-B-pc assign such an alternative $a$ to the highest category $C^{\ell}$ for which $a I_{\lambda} \pi^{\ell}$. With our proposal, the alternative $a$ is assigned by ETRI-B-dpc (the same is true for ETRI-B-dpd) to the lowest category $C^{k}$ for which $a I_{\lambda} \pi^{k+1}$.

Still interpreting ETRI-B as the conjoint use of ETRI-B-pc and ETRI-B-pd, each alternative is assigned to an interval of categories. Our proposal does the same thing. The interval obtained with our proposal is always "wider" than the one obtained with the original method. This is detailed in Table 10. Hence, our proposal may be seen as more "cautious" than the original method.

Let us finally notice that, in ETRI-B, an alternative is assigned by ETRI-B-pc, to a category that is never higher than the category to which it is assigned by ETRI-B-pd. This is no more the case with our proposal (see Table 10). While, when an alternative is incomparable to a set of consecutive profiles, the assignment with ETRI-B-pc is always lower than the assignment with ETRI-B-dpc, the situation is now reversed when an alternative is indifferent to several consecutive profiles.

\begin{tabular}{ccccccc}
\hline & \multicolumn{3}{c}{ Original method } & \multicolumn{3}{c}{ Our proposal } \\
& ETRI-B-pc & ETRI-B-pd & Interval & ETRI-B-pc & ETRI-B-dpc & Interval \\
\hline 1 & $k_{1}$ & $k_{1}$ & {$\left[k_{1}, k_{1}\right]$} & $k_{1}$ & $k_{1}$ & {$\left[k_{1}, k_{1}\right]$} \\
2 & $\ell_{2}$ & $\ell_{2}$ & {$\left[\ell_{2}, \ell_{2}\right]$} & $\ell_{2}$ & $k_{2}$ & {$\left[k_{2}, \ell_{2}\right]$} \\
3 & $k_{3}$ & $\ell_{3}$ & {$\left[k_{3}, \ell_{3}\right]$} & $k_{3}$ & $\ell_{3}$ & {$\left[k_{3}, \ell_{3}\right]$} \\
\hline
\end{tabular}

Table 10: Comparison of the conjoint use of ETRI-B-pc and ETRI-B-pd with the conjoint use of ETRI-B-pc and ETRI-B-dpc. The three cases refer to Table 5.

Our proposal has advantages and disadvantages. We analyze them below.

\subsubsection{Limitations}

Our proposal rests on a conception of limiting profiles that is different from the original one. Profiles are here at the frontier between two categories and may belong to each of them (we have seen that this is also the case with the conjunctive and disjunctive rules). Hence, it cannot be excluded that this conception of profiles will be seen as more complex than the original one by some decision makers. If this is the case, this could make more difficult a direct elicitation of the profiles from the decision maker.

Although most of the recent literature has favored indirect over direct elicitation techniques (see, e.g., Kadziński, Greco, and Słowiński, 2012, Słowiński, Greco, and Matarazzo, 2005) ${ }^{4}$, this potential drawback would remain since we do not want to a priori exclude such a direct elicitation and we do not have at hand an indirect elicitation technique that would elicit profiles for the variant that we suggest ${ }^{5}$. The conception of such indirect elicitation techniques for this variant is clearly an

\footnotetext{
${ }^{4}$ A few quotes may be useful at that point: "Very often in multicriteria decision analysis, this information has to be given in terms of preference model parameters, such as importance weights, substitution ratios and various thresholds. Presenting such information requires significant effort on the part of the decision maker. It is generally acknowledged that people often prefer to make exemplary decisions and cannot always explain them in terms of specific parameters. For this reason, the idea of inferring preference models from exemplary decisions provided by the decision maker is very attractive." (Słowiński et al., 2005, p. 477) or "When it comes to preference information, it may be either direct or indirect, depending on whether it specifies directly values of some parameters used in the preference model, or if it specifies some examples of holistic judgments. Presently, methods requiring the indirect preference information become more and more popular. It is the case because they require from DMs to exercise their decisions rather than to explain them in terms of parameters of a preference model. This way is natural for the DM and requires little cognitive effort. On the other hand, direct preference information can originate misleading answers, because the real meaning of concepts such as, e.g., importance weights or comparison thresholds could be unclear for the DM." (Kadziński et al., 2012 , p. 488).

${ }^{5}$ Clearly elicitation techniques that exist for ETRI-B-pc can be applied for ETRI-B-dpc without any change. We mean here that we do not have elicitation techniques that would allow to find the parameters of the variant that we
} 
important topic for future research.

\subsubsection{Advantages}

Let us now try to explain why our proposal may be of some interest. Our main argument is that it deals with the features presented in Section 4.1 more in accordance with our intuition than does ETRI-B.

Consider first an alternative $a$ such that $\pi^{k+1} I_{\lambda} a$ and $\pi^{k+1} \Delta^{*} a \Delta^{*} \pi^{k}$. Suppose furthermore, for simplicity, that $a$ is only indifferent to the profile $\pi^{k+1}$. We have seen above that, with the preference model used in ETRI-B, this case may happen and is not rare. Such an alternative is assigned to $C^{k+1}$ by both ETRI-B-pc and ETRI-B-pd. Although this is a direct implication of the second part of the conformity principle, we find it difficult to accept since, in our view, the assignment of $a$ to $C^{k}$ should not be discarded. This is precisely what happens with our proposal. With ETRI-B-pc, such an alternative will be assigned to $C^{k+1}$. However, using ETRI-B-dpc, it will be assigned to $C^{k}$.

Second, our proposal gives two versions that correspond via the transposition operation (see Table 8). Hence, when using our proposal, exactly similar results are obtained with the original and with the transposed problem. We have seen that this is not the case with ETRI-B.

Third, our proposal contains as particular cases the conjunctive and disjunctive rules, whereas we have seen that this is not the case with ETRI-B. When indifference and preference thresholds are null and $\lambda=1$, ETRI-B-pc is exactly the conjunctive rule and ETRI-B-dpc is exactly the disjunctive rule.

Fourth, our proposal is entirely based on $S_{\lambda}$ and makes no use of $P_{\lambda}$. As discussed above, we view this fact as an advantage (in view of the analysis in Bouyssou and Pirlot, 2013, 2014) since the preference model used in ETRI-B focuses on $S_{\lambda}$ instead of $P_{\lambda}$ (for an outranking method concentrating on strict preference, see Vansnick, 1986. This method has been analyzed from an axiomatic point of view in Bouyssou and Vansnick, 1986, and Bouyssou and Pirlot, 2012).

Fifth, the axiomatic analysis conducted in Bouyssou and Marchant (2007a,b) for ETRI-B-pc applies without any change to ETRI-B-dpc. This is obvious since using ETRI-B-dpc is equivalent to applying ETRI-B-pc to the transposed problem. This shows, in our view, that the two variants used in our proposal share the same underlying principles. Following the analysis in Bouyssou and Marchant (2007a, p. 228, Example 14), we know that this is not the case with ETRI-B. While ETRI-B-pc fits into the framework of "noncompensatory sorting models" (Bouyssou and Marchant, 2007a, p. 226 and 235), ETRI-B-pd does not. Let us finally observe that the framework of "noncompensatory sorting models" seems to be quite well adapted to the development of elicitation techniques (see, e.g., Cailloux et al., 2012, Leroy et al., 2011, and the comments in Zheng et al., 2014).

\section{Discussion}

This paper has analyzed several aspects of ELECTRE TRI, triggered by the recent proposal of ELECTRE TRI-C.

We have first shown that the relations between ETRI-B and ETRI-C are complex. Indeed, we have shown that there are ordered partitions that can be obtained with ETRI-B and that cannot be obtained with ETRI-C and vice versa. We view this fact as the sign that the situation with central profiles is less similar to the situation with limiting profiles as one might think (Figueira, 2013 and Roy, 2013, reacting to a previous version of this text, stress this point). This clearly calls for further research. In particular, three points would deserve further work. First, it would be nice to know whether it is possible to propose a method working with central profiles that would have simpler relations with ETRI-B, in the sense explored in Section 3. Second, it would be interesting to

suggest in which ETRI-B-pc and ETRI-B-dpc are use conjointly. 
investigate the theoretical properties of ETRI-C, mimicking what Bouyssou and Marchant (2007a,b) have done with ETRI-B. Finally, ETRI-C has been introduced without the proposal of specific elicitation techniques. This is clearly a crucial direction for future research (see Almeida-Dias, 2011, Ch. 7, for preliminary results on the subject).

Second, motivated by a number of features exhibited by ETRI-B that do not fit well our intuition, we have proposed a simple variant of the original method. This variant abandons the second part of the conformity requirement introduced in Roy and Bouyssou (1993). This implies a more complex interpretation of profiles that are now seen as frontiers between categories. With this proposal, each alternative is assigned to an interval of consecutive categories. The results obtained are not affected by the transposition operation. The interest and usefulness of this variant should clearly be analyzed further. Our analysis nevertheless already shows that it may have some advantages, compared to the original ELECTRE TRI-B. Indeed, it offers a simple and yet clear solution to the features of ETRI-B that did not fit well our intuition, while remaining in line with the original method.

\section{References}

J. Almeida-Dias. Multiple criteria decision aiding for sorting problems: Concepts, methodologies, and applications. PhD dissertation, Universidade Téchnica de Lisboa and Université Paris Dauphine, Lisbon and Paris, 2011.

J. Almeida-Dias, J. R. Figueira, and B. Roy. ELECTRE TRI-C: A multiple criteria sorting method based on characteristic reference actions. European Journal of Operational Research, 204(3):565-580, 2010.

J. Almeida-Dias, J. R. Figueira, and B. Roy. A multiple criteria sorting method where each category is characterized by several reference actions: The ELECTRE TRI-nC method. European Journal of Operational Research, 217(3):567-579, 2012.

D. Bouyssou. Outranking relations: Do they have special properties? Journal of Multi-Criteria Decision Analysis, 5(2):99-111, 1996.

D. Bouyssou and T. Marchant. An axiomatic approach to noncompensatory sorting methods in MCDM, I:

The case of two categories. European Journal of Operational Research, 178(1):217-245, 2007a.

D. Bouyssou and T. Marchant. An axiomatic approach to noncompensatory sorting methods in MCDM, II: More than two categories. European Journal of Operational Research, 178(1):246-276, 2007b.

D. Bouyssou and M. Pirlot. Analyzing the correspondence between strict and non-strict outranking relations. Communication to EURO XXIII, Bonn, July 2009, EURO, 2009.

D. Bouyssou and M. Pirlot. An axiomatic approach to TACTIC. Studia Informatica Universalis, 10(2):45-71, 2012.

D. Bouyssou and M. Pirlot. On the relationship between strict and non-strict outranking relations. Cahiers du LAMSADE 346, LAMSADE, Université Paris Dauphine, November 2013.

D. Bouyssou and M. Pirlot. A note on the asymmetric part of an outranking relation. Cahiers du LAMSADE 351, LAMSADE, Université Paris Dauphine, March 2014.

D. Bouyssou and J.-C. Vansnick. Noncompensatory and generalized noncompensatory preference structures. Theory and Decision, 21(3):251-266, 1986.

O. Cailloux, P. Meyer, and V. Mousseau. Eliciting ELECTRE TRI category limits for a group of decision makers. European Journal of Operational Research, 223(1):133-140, 2012.

S. Damart, L. C. Dias, and V. Mousseau. Supporting groups in sorting decisions: Methodology and use of a multi-criteria aggregation / disaggregation DSS. Decision Support Systems, 43(4):1464-1475, 2007.

L. C. Dias and J. Clímaco. ELECTRE TRI for groups with imprecise information on parameter values. Group Decision and Negotiation, 9(5):355-377, 2000.

L. C. Dias and V. Mousseau. IRIS: A DSS for multiple criteria sorting problems. Journal of Multi-Criteria Decision Analysis, 12:285-298, 2003.

L. C. Dias and V. Mousseau. Inferring ELECTRE's veto-related parameters from outranking examples. European Journal of Operational Research, 170(1):172-191, 2006.

L. C. Dias, V. Mousseau, J. Figueira, and J. Clímaco. An aggregation/disaggregation approach to obtain robust conclusions with ELECTRE TRI. European Journal of Operational Research, 138(2):332-48, 2002. 
J. R. Figueira. Personal communication to the authors, 16 September 2013.

J. R. Figueira, V. Mousseau, and B. Roy. ELECTRE methods. In J. R. Figueira, S. Greco, and M. Ehrgott, editors, Multiple Criteria Decision Analysis: State of the Art Surveys, number 28 in International Series in Operations Research \& Management Science, pages 133-162. Springer Verlag, Boston, Dordrecht, London, 2005. ISBN 0-387-23067-X.

J. R. Figueira, S. Greco, B. Roy, and R. Słowiński. An overview of ELECTRE methods and their recent extensions. Journal of Multi-Criteria Decision Analysis, 20(1-2):61-85, 2013.

S. Greco, V. Mousseau, and R. Słowiński. Multiple criteria sorting with a set of additive value functionss. European Journal of Operational Research, 207(3):1455-1470, 2010.

É. Jacquet-Lagrèze. An application of the UTA discriminant model for the evaluation of R\&D projects. In P. Pardalos, Y. Siskos, and C. Zopounidis, editors, Advances in Multicriteria Analysis, pages $203-211$. Kluwer, Dordrecht, 1995.

M. Kadziński, S. Greco, and R. Słowiński. Extreme ranking analysis in robust ordinal regression. Omega, 40: 488-501, 2012.

A. Leroy, V. Mousseau, and M. Pirlot. Learning the parameters of a multiple criteria sorting method. In R. I. Brafman, F. S. Roberts, and A. Tsoukiàs, editors, ADT 2011, number 6992 in LNAI, pages 219-233. Springer, 2011.

V. Mousseau and L. C. Dias. Valued outranking relations in ELECTRE providing manageable disaggregation procedures. European Journal of Operational Research, 156(2):467-482, 2004.

V. Mousseau and R. Słowiński. Inferring an ELECTRE TRI model from assignment examples. Journal of Global Optimization, 12(2):157-174, 1998.

V. Mousseau, R. Słowiński, and P. Zielniewicz. A user-oriented implementation of the ELECTRE TRI method integrating preference elicitation support. Computers $\&$ Operations Research, 27(7-8):757-777, 2000.

V. Mousseau, J. Figueira, and J.-P. Naux. Using assignment examples to infer weights for ELECTRE TRI method: Some experimental results. European Journal of Operational Research, 130(2):263-275, 2001.

V. Mousseau, J. R. Figueira, L. C. Dias, C. Gomes da Silva, and J. Clímaco. Resolving inconsistencies among constraints on the parameters of an MCDA model. European Journal of Operational Research, 147(1):72-93, 2003.

V. Mousseau, L. C. Dias, and J. R. Figueira. Dealing with inconsistent judgments in multiple criteria sorting models. 4OR, A Quarterly Journal of Operations Research, 4(2):147-158, 2006.

A. Ngo The and V. Mousseau. Using assignment examples to infer category limits for the ELECTRE TRI method. Journal of Multi-Criteria Decision Analysis, 11(1):29-43, 2002.

B. Roy. Présentation et interprétation de la méthode ELECTRE TRI pour affecter des zones dans des catégories de risque. Document du LAMSADE 124, LAMSADE, Université Paris Dauphine, Paris, France, March 2002. 25 pages.

B. Roy. Personal communication to the authors, 1 October 2013.

B. Roy and D. Bouyssou. Aide multicritère à la décision : méthodes et cas. Economica, Paris, 1993.

B. Roy and P. Vincke. Pseudo-orders: Definition, properties and numerical representation. Mathematical Social Sciences, 14(2):263-274, 1987.

R. Słowiński, S. Greco, and B. Matarazzo. Rough set based decision support. In E. K. Burke and G. Kendall, editors, Search Methodologies: Introductory Tutorials in Optimization and Decision Support Techniques, pages 475-527, New York, 2005. Springer.

J.-C. Vansnick. On the problems of weights in MCDM (the noncompensatory approach). European Journal of Operational Research, 24(2):288-294, 1986.

Y. Wei. Aide multicritère à la décision dans le cadre de la problématique du tri : concepts, méthodes et applications. Thèse de doctorat, Université Paris Dauphine, Paris, France, 1992. (in French).

J. Zheng. Preference Elicitation for reference based aggregation models: Algorithms and Procedures. PhD thesis, École Centrale, Paris, 2012.

J. Zheng, S. A. Metchebon Takougang, V. Mousseau, and M. Pirlot. Learning criteria weights of an optimistic ELECTRE TRI sorting rule. Computers \& Operations Research, 49:28-40, 2014.

C. Zopounidis and M. Doumpos. PREFDIS: A multicriteria decision support system for sorting decision problems. Computers $\&$ Operations Research, 27(7-8):779-797, 2000. 